\title{
Exploiting timing information in event-triggered stabilization of linear systems with disturbances
}

\author{
Mohammad Javad Khojasteh, Mojtaba Hedayatpour, Jorge Cortés, Massimo Franceschetti
}

\begin{abstract}
Similar to the way pauses are used in spoken language to convey information, it is also possible to transmit information in communication networks not only by message content, but also with its timing. This paper presents an eventtriggering strategy that utilizes timing information by transmitting in a state-dependent fashion. We consider the stabilization of a continuous-time, time-invariant, linear plant over a digital communication channel with bounded delay and subject to bounded plant disturbances and establish two main results. On the one hand, we design an encoding-decoding scheme that guarantees a sufficient information transmission rate for stabilization. On the other hand, we determine a lower bound on the information transmission rate necessary for stabilization by any control policy.
\end{abstract}

\section{INTRODUCTION}

In many networked control systems (NCS), the feedback loop is closed over a communication channel [1]. In this context, data-rate theorems [2], [3] state that the minimum communication rate to achieve stabilization is equal to the entropy rate of the plant, expressed by the sum of the unstable modes in nats (one nat corresponds to $1 / \ln 2$ bits.) Key contributions by [4], [5], and [6] consider a "bit-pipe" communication channel, capable of noiseless transmission of a finite number of bits per unit time evolution of the plant. Extensions to noisy communication channels are considered in [7]-[10]. Additional formulations include stabilization of switched linear systems [11], uncertain systems [12], nonlinear systems [13], [14], multiplicative noise [15], and optimal control [16], [17].

While the majority of communication networks transmit information by adjusting the content of the message, it is also possible to communicate information by adjusting the transmission time of a symbol [18]. In fact, it is known that event-triggering control techniques [19] encode information in timing in a state-dependent fashion [20]. The works [21]-[25] study event-triggered strategies over communication networks without exploiting the implicit timing information in the triggering events. The work [24] considers periodic eventtriggered control for linear systems where the event-triggering condition is verified periodically. The work [25] considers output feedback stabilization of linear systems with no disturbance where the measured outputs and control inputs are subject to event-triggered sampling and dynamic quantization.

M. J. Khojasteh is with the Center for Autonomous Systems and Technologies, California Institute of Technology. M. Hedayatpour is with the Faculty of Engineering and Applied Science, University of Regina, Canada. J. Cortés is with the Department of Mechanical and Aerospace Engineering, University of California, San Diego. M. Franceschetti is with the Department of Electrical and Computer Engineering of University of California, San Diego. (emails: mjkhojas@caltech.edu \{cortes, massimo\}@ucsd.edu, hedayatmeuregina.ca ). This research was partially supported by NSF awards CNS-1446891 and ECCS-1917177.
In contrast to the above works, to decrease the number of physical packets transmitted over the network (data payload), the works [20], [26]-[31] study event-triggered strategies that exploit the inherent timing information in the events, and show that stability can be achieved with a rate lower than the one prescribed by data-rate theorems. The work [26] utilizes the implicit timing information in triggering events to estimate a Wiener Process over a finite rate communication channel subject to finite delay. The work [27] uses eventtriggering to encode information in timing for stabilization of linear systems without disturbances in a silence-based communication manner [32]. Also, [33] extends the results of [27] to optimal control. The works [28], [29] show that, with sufficiently small delays, and assuming the controller has knowledge of the triggering strategy, one can stabilize the plant with any positive data payload transmission rate. These results are extended in [31] to a large class of triggering strategies. The work [30] provides a sufficient data payload rate for second-order systems with real eigenvalues. While in these works the delay is assumed to be sufficiently small to achieve stabilization, [20] considers arbitrary transmission delays in the communication network and quantifies the information contained in the timing of the events for the stabilization of scalar plants without disturbances. In [20] it is shown that for small delay values stability can be achieved with any positive information transmission rate (the rate at which sensor transmits data payload). However, as the delay increases to values larger than a critical threshold, the timing information contained in the triggering action itself may not be enough to stabilize the plant and the information transmission rate must be increased. The results in [20] are valid for vector plants when the open-loop gain matrix has only real eigenvalues.

The literature has not considered to what extent the implicit timing information in the triggering events is useful in the presence of plant disturbances for the whole spectrum of possible bounded communication delays. Beyond the uncertainty due to the unknown delay in communication, disturbances add an additional degree of uncertainty to the state estimation process. The required rate for stabilization and the viable notion for stabilization over communication channels critically depend on the presence of disturbances [5], [7], [8]. With this in mind, and in contrast to [20] that requires exponential convergence guarantees, here we study input-to-state practical stability (ISpS) [34], [35] of a linear, time-invariant plant subject to bounded disturbances over a communication channel with arbitrarily large but bounded delay.

Our contributions are threefold. First, for scalar real plants with disturbances, we derive a sufficient condition on the information transmission rate for the whole spectrum of pos- 
sible communication delay values. Specifically, we design an encoding-decoding scheme that, together with the proposed event-triggering strategy, rules out Zeno behavior and ensures that there exists a control policy which renders the plant ISpS. We show that for small values of the delay, our event-triggering strategy achieves ISpS using only implicit timing information and transmitting data payload at a rate arbitrarily close to zero. On the other hand, since larger values of the delay imply that the information transmitted has become excessively outdated and corrupted by the disturbance, increasingly higher communication rates are required as the delay becomes larger. Our second contribution pertains to the generalization of the sufficient condition to complex plants with complex openloop gain subject to disturbances. This result sets the basis for the generalization of event-triggered control strategies that meet the bounds on the information transmission rate for the ISpS of vector systems under disturbances and with any real open-loop gain matrix (with complex eigenvalues). The first two contributions provide stronger results than our preliminary conference papers [36], [37] and contain a complete technical treatment. Our final contribution is a necessary condition on the information transmission rate for scalar real plants, assuming that at each triggering time the sensor transmits the smallest possible packet size to achieve the triggering goal for all realizations of the delay and plant disturbance. The simulation results are presented in Appendix A.

\section{PROBLEM FORMULATION}

We consider ${ }^{1}$ a NCS described by a plant-sensor-channelcontroller tuple, cf. Fig. 1. The plant is described by a scalar, continuous-time, linear time-invariant model,

$$
\dot{x}=A x(t)+B u(t)+w(t),
$$

where $x(t) \in \mathbb{R}$ and $u(t) \in \mathbb{R}$ for $t \in[0, \infty)$ are the plant state and control input, respectively, and $w(t) \in \mathbb{R}$ represents

\footnotetext{
${ }^{1}$ Throughout the paper, we use the following notation. $\mathbb{R}, \mathbb{R}_{\geq 0}, \mathbb{C}$, and $\mathbb{N}$ represent the set of real, nonnegative real, complex, and natural numbers, resp. We let $|$.$| and \|$.$\| denote absolute value and complex absolute value, resp.$ Let $\log$ and $\ln$ represent base 2 and natural logarithms, resp. For a function $f: \mathbb{R} \rightarrow \mathbb{R}^{n}$ and $t \in \mathbb{R}$, we let $f\left(t^{+}\right)=\lim _{s \rightarrow t^{+}} f(s)$ denote the righthand limit of $f$ at $t$. In addition, $\lfloor x\rfloor$ (resp. $\lceil x\rceil$ ) denotes the nearest integer less (resp. greater) than or equal to $x$. We denote the modulo function by $\bmod (x, y)$, representing the remainder after division of $x$ by $y$. The function $\operatorname{sign}(x)$ denotes the sign of $x$. Any $Q \in \mathbb{C}$ can be written as $Q=\operatorname{Re}(Q)+$ $i \operatorname{Im}(Q)=\|Q\| e^{i \phi_{Q}}$, and for any $y \in \mathbb{R}$ we have $\left\|e^{Q y}\right\|=e^{\operatorname{Re}(Q) y}$ $\operatorname{Tr}(A)$ denotes the trace of matrix $A$, and $m$ denotes the Lebesgue measure. For a scalar continuous-time signal $w(t)$, we define $|w|_{t}=\sup _{s \in[0, t]}|w(s)|$. To formulate the stability properties, for non-negative constant $d$ we define

$$
\begin{gathered}
\mathcal{K}(d):=\left\{f: \mathbb{R}_{\geq 0} \rightarrow \mathbb{R}_{\geq 0} \mid f\right. \text { continuous, } \\
\text { strictly increasing, and } f(0)=d\}, \\
\mathcal{K}_{\infty}(d):=\{f \in \mathcal{K}(d) \mid f \text { unbounded }\}, \\
\mathcal{K}_{\infty}^{2}:=\left\{f: \mathbb{R}_{\geq 0} \times \mathbb{R}_{\geq 0} \rightarrow \mathbb{R}_{\geq 0} \mid \forall t>0,\right. \\
\left.f(., t) \in \mathcal{K}_{\infty}(0), \text { and } \forall r>0 f(r, .) \in \mathcal{K}_{\infty}(0)\right\} \\
\mathcal{L}:=\left\{f: \mathbb{R}_{\geq 0} \rightarrow \mathbb{R}_{\geq 0} \mid f\right. \text { continuous, } \\
\left.\quad \text { strictly decreasing, and } \lim _{s \rightarrow \infty} f(s)=0\right\}, \\
\mathcal{K} \mathcal{L}:=\left\{f: \mathbb{R}_{\geq 0} \times \mathbb{R}_{\geq 0} \rightarrow \mathbb{R}_{\geq 0} \mid f\right. \text { continuous, } \\
\forall t \geq 0, f(., t) \in \mathcal{K}(0), \text { and } \forall r>0 f(r, .) \in \mathcal{L}\} .
\end{gathered}
$$
}

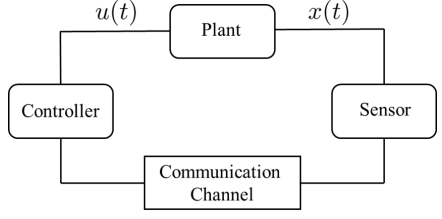

Fig. 1. Networked control system model.

the plant disturbance. The latter is a Lebesgue-measurable function of time, and upper bounded as

$$
|w(t)| \leq M
$$

where $M \in \mathbb{R}_{\geq 0}$. In (1), $A \in \mathbb{R}$ is positive (i.e., the plant is unstable), $B \in \mathbb{R} \backslash\{0\}$, and the initial condition $x(0)$ is bounded. We assume the sensor measurements are exact and there is no delay in the control action, which is executed with infinite precision. However, measurements are transmitted from sensor to controller over a communication channel subject to a finite data rate and bounded unknown delay $^{2}$. We denote by $\left\{t_{s}^{k}\right\}_{k \in \mathbb{N}}$ the sequence of times when the sensor transmits a packet of length $g\left(t_{s}^{k}\right)$ bits containing a quantized version of the encoded state. We let $\Delta_{k}^{\prime}=t_{s}^{k+1}-t_{s}^{k}$ be the $k^{t h}$ triggering interval. The packets are delivered to the controller without error and entirely but with unknown upper bounded delay. Let $\left\{t_{c}^{k}\right\}_{k \in \mathbb{N}}$ be the sequence of times where the controller receives the packets transmitted at times $\left\{t_{s}^{k}\right\}_{k \in \mathbb{N}}$. We assume the communication delays $\Delta_{k}=t_{c}^{k}-t_{s}^{k}$, for all $k \in \mathbb{N}$, satisfy

$$
\Delta_{k} \leq \gamma,
$$

where $\gamma \in \mathbb{R}_{\geq 0}$. When referring to a generic triggering or reception time, for convenience, we skip the super-script $k$ in $t_{s}^{k}$ and $t_{c}^{k}$, and the sub-script $k$ in $\Delta_{k}$ and $\Delta_{k}^{\prime}$. In our model, clocks are synchronized at the sensor and the controller. In case of using a timestamp, due to the communication constraints, only a quantized version of it can be encoded in the packet $g\left(t_{s}\right)$.

At the controller, the estimated state is represented by $\hat{x}$ and evolves during the inter-reception times as

$$
\dot{\hat{x}}(t)=A \hat{x}(t)+B u(t), \quad t \in\left(t_{c}^{k}, t_{c}^{k+1}\right),
$$

starting from $\hat{x}\left(t_{c}^{k+}\right)$, which represents the state estimate of the controller with the information received up to time $t_{c}^{k}$ with initial condition $\hat{x}(0)$ (the exact way to construct $\hat{x}\left(t_{c}^{k+}\right)$ is explained later in Section III).

Assumption 1: The sensor can compute $\hat{x}\left(t_{c}^{k+}\right)$ for all $k \in$ $\mathbb{N}$.

Remark 1: We show in Proposition 2 that Assumption 1 is valid for our controller design, provided the sensor knows the times the actuator performs the control action. This is a common practice in TCP-based networks, where packet arrivals are acknowledged via a communication feedback link, to ensure the robust transmission of the packets, see e.g. [39][41]. In NCS, this corresponds to assuming an instantaneous

\footnotetext{
${ }^{2}$ In general, there might also be a communication channel with finite capacity in the downlink, between the controller and the plant. However, in many applications such as mobile robots [38], the uplink, which is studied here, is the main bottleneck, as a strong on-board transmitter reduces the operating duration, restricts robot mobility, and increases cost.
} 
acknowledgment from the actuator to the sensor via the control input, known as communication through the control input [7], [8], [42]. To obtain such causal knowledge, one can monitor the output of the actuator provided that the control input changes at each reception time. In case the sensor has only access to the plant state, since the system disturbance is bounded (2), assuming that the control input is continuous during inter-reception times and jumps in the reception times such that $B\left|u\left(t_{c}\right)-u\left(t_{c}^{-}\right)\right|>M$, the controller can signal the reception time of the packet to the sensor via $\dot{x}(t)$. Finally, we note that any necessary condition on the information transmission rate obtained with Assumption 1 in place remains necessary without it as well (cf. Section IV-B).

Under Assumption 1, the sensor can use (4) to compute $\hat{x}(t)$ for all $t \geq 0$, provided it knows $\hat{x}(0)$. Thus, under this assumption, the estimation error at the sensor is

$$
z(t)=x(t)-\hat{x}(t),
$$

and we rely on this error to determine when a triggering event occurs in our controller design. We next define a modified version of input-to-state practical stability (ISpS) [34], [35], which is suitable for the present setup.

Definition 1: The plant (1) is ISpS if there exist $\xi \in \mathcal{K} \mathcal{L}$, $\psi \in \mathcal{K}_{\infty}(0), d \in \mathbb{R}_{\geq 0}, \iota \in \mathcal{K}_{\infty}(d)$, and $\vartheta \in \mathcal{K}_{\infty}^{2}$ such that

$$
|x(t)| \leq \xi(|x(0)|, t)+\psi\left(|w|_{t}\right)+\iota(\gamma)+\vartheta\left(|w|_{t}, \gamma\right), \forall t \geq 0 .
$$

Note that, for a fixed $\gamma$, this definition reduces to the standard notion of ISpS. Given that the initial condition, delay, and system disturbances are bounded, ISpS implies that the state must be bounded at all times. Our objective is to ensure the dynamics (1) is ISpS given the constraints posed by the system model of Fig. 1. Let $b_{s}(t)$ be the number of bits transmitted in the data payload by the sensor up to time $t$. The information transmission rate is

$$
R_{s}=\limsup _{t \rightarrow \infty}\left(b_{s}(t) / t\right)=\limsup _{N \rightarrow \infty}\left(\sum_{k=1}^{N} g\left(t_{s}^{k}\right) / \sum_{k=1}^{N} \Delta_{k}^{\prime}\right),
$$

where the latter equality follows by noting that, at each triggering time $t_{s}^{k}$, the sensor transmits $g\left(t_{s}^{k}\right)$ bits.

In addition to the data payload, the reception time of the packets carries information. Consequently, let $b_{c}(t)$ be the amount of information measured in bits included in data payload and timing information received at the controller until time $t$. The information access rate is $R_{c}=$ $\lim \sup _{t \rightarrow \infty}\left(b_{c}(t) / t\right)$.

Remark 2: We do not consider the bounded delays (3) to be chosen from any specific distribution. Thus, the information that can be gained about the triggering time $t_{s}$ from the reception time $t_{c}$ may be quantified by the Rényi 0th-order information functional $I_{0}$ [43], [44]. Assuming the controller has received $N$ packet by time $t$, we deduce $b_{c}(t)=\sum_{k=1}^{N}\left(g\left(t_{s}^{k}\right)+I_{0}\left(t_{s}^{k} ; t_{c}^{k}\right)\right)$.

According to the data-rate theorem [20], [45], if $R_{c}<$ $A / \ln 2$, the value of the state in (1) becomes unbounded as $t \rightarrow \infty$, and hence (1) is not ISpS. The data-rate theorem characterizes what is needed by the controller, and does not depend on the specific feedback structure (including aspects such as information pattern at the sensor/controller, commu- nication delays, and whether transmission times are statedependent, as in event-triggered control, or periodic, as in time-triggered control). In our discussion below, the bound $R_{c}=A / \ln 2$ serves as a baseline for our results on the information transmission rate $R_{s}$ to understand the amount of timing information contained in event-triggered control designs in the presence of unknown communication delays.

We do not consider delays, plant disturbances, and initial condition to be chosen from any specific distribution. Therefore, our results are valid for any arbitrary delay, plant disturbances, and initial condition with finite support. In particular, our goal is to find upper and lower bounds on $R_{s}$, where the lower bound is necessary at least for a realization of the initial condition, delay, and disturbances, and the upper bound is sufficient for all realizations of the initial condition, delay, and disturbances. In addition, our lower bound is necessary for any control policy $u(t)$ to render the plant (1) ISpS under the class of event-triggering strategies described next.

\section{EVENT-TRIGGERED DESIGN}

Here we introduce the general class of event-triggered policies considered in this paper. Consider the following class of triggers: for $J \in \mathbb{R}$ positive, the sensor sends a message to the controller at $t_{s}^{k+1}$ if

$$
\left|z\left(t_{s}^{k+1}\right)\right|=J
$$

provided $t_{c}^{k} \leq t_{s}^{k+1}$ for $k \in \mathbb{N}$ and $t_{s}^{1} \geq 0$. A new transmission happens only after the previous packet has been received by the controller. Since the triggering time $t_{s}$ is a real number, its knowledge can reveal an unbounded amount of information to the controller. However, due to the unknown delay in the communication network, the controller does not have perfect knowledge of it. In fact, both the finite data rate and the delay mean that the controller may not be able to compute the exact value of $x\left(t_{c}\right)$. To address this, let $\bar{z}\left(t_{c}\right)$ be an estimated version of $z\left(t_{c}\right)$ reconstructed by the controller knowing $\left|z\left(t_{s}\right)\right|=J$, the bound (3) on the delay, and the packet received through the channel. Using $\bar{z}\left(t_{c}\right)$, the controller updates the state estimate via the jump strategy,

$$
\hat{x}\left(t_{c}^{+}\right)=\bar{z}\left(t_{c}\right)+\hat{x}\left(t_{c}\right) \text {. }
$$

Note that $\left|z\left(t_{c}^{+}\right)\right|=\left|x\left(t_{c}\right)-\hat{x}\left(t_{c}^{+}\right)\right|=\left|z\left(t_{c}\right)-\bar{z}\left(t_{c}\right)\right|$. We assume the packet size $g\left(t_{s}\right)$ calculated at the sensor is so that

$$
\left|z\left(t_{c}^{+}\right)\right|=\left|z\left(t_{c}\right)-\bar{z}\left(t_{c}\right)\right| \leq J
$$

is satisfied for all $t_{c} \in\left[t_{s}, t_{s}+\gamma\right]$. This property is key in our forthcoming developments. In particular, we will show that our controller design for the sufficient characterization on the transmission rate is based on identifying a particular encodingdecoding strategy and a packet size to ensure (9). Likewise, our necessary characterization is based on identifying the minimal packet sizes necessary to ensure (9).

The importance of (9) starts to become apparent in the following result: if this inequality holds at each reception time, the state estimation error (5) is bounded for all time.

Lemma 1: Consider the model with plant dynamics (1), estimator dynamics (4), triggering strategy (7), and jump 
strategy (8). Assume $|z(0)|=|x(0)-\hat{x}(0)|<J$ and (9) holds at all reception times $\left\{t_{c}^{k}\right\}_{k \in \mathbb{N}}$. Then, for all $t \geq 0$,

$$
|z(t)| \leq J e^{A \gamma}+\frac{|w|_{t}}{A}\left(e^{A \gamma}-1\right) .
$$

Proof: At the reception time, $z\left(t_{c}^{k+}\right)$ satisfies (9), hence using the triggering rule (7), we deduce $|z(t)| \leq J$ for all $t \in\left(t_{c}^{k}, t_{s}^{k+1}\right]$. Since $J$ is smaller than the upper bound in (10), and $z\left(t_{c}^{(k+1)+}\right)$ satisfies (9), it remains to prove (10) for $t \in\left(t_{s}^{k+1}, t_{c}^{k+1}\right)$. From (1), (4), and (5), we have $\dot{z}(t)=A z(t)+w(t)$ during inter-reception time intervals $\left(t_{c}^{k}, t_{c}^{k+1}\right)$. Also, from (7) it follows $\left(t_{s}^{k+1}, t_{c}^{k+1}\right) \subseteq\left(t_{c}^{k}, t_{c}^{k+1}\right)$. Thus, for all $t \in\left(t_{s}^{k+1}, t_{c}^{k+1}\right)$, we have

$$
z(t)=e^{A\left(t-t_{s}^{k+1}\right)} z\left(t_{s}^{k+1}\right)+\int_{t_{s}^{k+1}}^{t} e^{A(t-\tau)} w(\tau) d \tau .
$$

When a triggering occurs $\left|z\left(t_{s}^{k+1}\right)\right|=J$, hence the absolute value of the first addend in (11) is upper bounded by $J e^{A\left(t-t_{s}^{k+1}\right)}$. Also, for the second addend in (11) we have

$$
\begin{aligned}
& \quad\left|\int_{t_{s}^{k+1}}^{t} e^{A(t-\tau)} w(\tau) d \tau\right| \\
& \quad \leq|w|_{t} \int_{t_{s}^{k+1}}^{t}\left|e^{A(t-\tau)}\right| d \tau=\frac{|w|_{t}}{A}\left(e^{A\left(t-t_{s}^{k+1}\right)}-1\right) .
\end{aligned}
$$

By (3), $t-t_{s}^{k+1} \leq t_{c}^{k+1}-t_{s}^{k+1} \leq \gamma$, and the result follows.

We continue by showing that, if (9) holds at each reception time $\left\{t_{c}^{k}\right\}_{k \in \mathbb{N}}$, then a linear controller renders the plant (1) ISpS. We note that similar results exist in the literature (e.g., [24], [46], [47]) and we extend them here to our eventtriggering setup with quantization and unknown delays.

Proposition 1: Under the assumptions of Lemma 1, the controller $u(t)=-K \hat{x}(t)$ renders (1) ISpS, provided $A-$ $B K<0$.

Proof: By letting $u(t)=-K(x(t)-z(t))$, we rewrite (1) as $\dot{x}(t)=(A-B K) x(t)+B K z(t)+w(t)$. Consequently,

$$
\begin{aligned}
|x(t)| \leq & e^{(A-B K) t}|x(0)| \\
& +e^{(A-B K) t} \int_{0}^{t} e^{-(A-B K) \tau}(B K|z(\tau)|+|w(\tau)|) d \tau .
\end{aligned}
$$

since $A-B K<0$, the first summand in (13) is a $\mathcal{K} \mathcal{L}$ function of $|x(0)|$ and time. Thus, it remains to prove the second summand in (13) is upper bounded by summation of a $\mathcal{K}_{\infty}(0)$ function of $|w|_{t}$, a $\mathcal{K}_{\infty}(d)$ function of $\gamma$, and a $\mathcal{K}_{\infty}^{2}$ function of $|w|_{t}$ and $\gamma$. The second summand in (13) is upper bounded by $-\left(1-e^{(A-B K) t}\right)\left(B K|z|_{t}+|w|_{t}\right) /(A-B K)$. Since $1-e^{(A-B K) t}<1$, using Lemma 1 we deduce the second summand in (13) is upper bounded by $\psi\left(|w|_{t}\right)+\iota(\gamma)+$ $\vartheta\left(|w|_{t}, \gamma\right)$, where $\psi\left(|w|_{t}\right)=\left(|w|_{t} /-(A-B K)\right)$ which is a $\mathcal{K}_{\infty}(0)$ function of $|w|_{t}, \iota(\gamma)=\left(\left(B K J e^{A \gamma}\right) /-(A-B K)\right)$ which is a $\mathcal{K}_{\infty}(d)$ function of $\gamma$ with $d=\iota(0)$, and $\vartheta\left(|w|_{t}, \gamma\right)=\left(\left(B K|w|_{t}\right) /-A(A-B K)\right)\left(e^{A \gamma}-1\right)$ which is a $\mathcal{K}_{\infty}^{2}$ function of $\gamma$ and $|w|_{t}$.

Using (2), we deduce from Lemma 1 that $|z(t)| \leq J e^{A \gamma}+$ $\frac{M}{A}\left(e^{A \gamma}-1\right)$ for all $t \geq 0$. Next, we rule out Zeno behavior (an infinite amount of events in a finite time interval) for our event-triggered control design. To do this, let $0<\rho_{0}<1$ be a design parameter, and assume the packet size $g\left(t_{s}\right)$ is selected at the sensor to ensure a stronger version of (9),

$$
\left|z\left(t_{c}^{+}\right)\right|=\left|z\left(t_{c}\right)-\bar{z}\left(t_{c}\right)\right| \leq \rho_{0} J .
$$

Clearly, (14) implies (9). Next, we show that given (14), the time between consecutive triggers is uniformly lower bounded.

Lemma 2: Consider the model with plant dynamics (1), estimator dynamics (4), triggering strategy (7), and jump strategy (8). Assume $|z(0)|=|x(0)-\hat{x}(0)|<J$ and (14) holds at all reception times $\left\{t_{c}^{k}\right\}_{k \in \mathbb{N}}$. Then for all $k \in \mathbb{N}$ $t_{s}^{k+1}-t_{s}^{k} \geq \ln \left(\frac{J A+M}{\rho_{0} J A+M}\right) / A$.

Proof: By considering two successive triggering times $t_{s}^{k}$ and $t_{s}^{k+1}$ and the reception time $t_{c}^{k}$, from (7) it follows $t_{s}^{k} \leq t_{c}^{k} \leq t_{s}^{k+1}$. From (1), (4), and (5), we have $\dot{z}(t)=A z(t)+w(t)$ during inter-reception time intervals $\left(t_{c}^{k}, t_{c}^{k+1}\right)$, consequently using the definition of the triggering time $t_{s}^{k+1}$ (7) it follows $\left|z\left(t_{c}^{k+}\right) e^{A\left(t_{s}^{k+1}-t_{c}^{k}\right)}\right|+$ $\left|\int_{t_{c}^{k}}^{t_{s}^{k+1}} e^{A\left(t_{s}^{k+1}-\tau\right)} w(\tau) d \tau\right| \geq J$. Using (14) and (12), we have $\rho_{0} J e^{A\left(t_{s}^{k+1}-t_{c}^{k}\right)}+(M / A)\left(e^{A\left(t_{s}^{k+1}-t_{c}^{k}\right)}-1\right) \geq J$, which is equivalent to $t_{s}^{k+1}-t_{c}^{k} \geq \frac{1}{A} \ln \left(\frac{J+\frac{M}{A}}{\rho_{0} J+\frac{M}{A}}\right)$. The result follows from using $t_{s}^{k} \leq t_{c}^{k}$ in this inequality.

Given the uniform lower bound on the inter-event time in Lemma 2, we deduce that the event-triggered control design does not exhibit Zeno behavior. The frequency of transmission events is captured by the triggering rate

$$
R_{t r}=\limsup _{N \rightarrow \infty}\left(N / \sum_{k=1}^{N} \Delta_{k}^{\prime}\right) .
$$

Using Lemma 2, we deduce that the triggering rate (15) is uniformly upper bounded under the event-triggered control design, i.e., for all initial conditions, possible delay and plant noise values,

$$
R_{t r} \leq A / \ln \left(\frac{J A+M A}{\rho_{0} J A+M}\right) .
$$

\section{SUFFICIENT AND NECESSARY CONDITIONS ON THE INFORMATION TRANSMISSION RATE}

Here we derive sufficient and necessary conditions on the information transmission rate (6) to ensure (1) is ISpS. Our approach is based on the characterization of the transmission rate required to ensure that (9) holds at all reception times. Section IV-A introduces a quantization policy that, together with the event-triggered scheme, provides a complete control design to guarantee (1) is ISpS and rules out Zeno behavior. Section IV-B presents lower bounds on the packet size and triggering rate required to guarantee (1) is ISpS, leading to our bound on the necessary information transmission rate.

\section{A. Sufficient information transmission rate}

1) Design of quantization policy: The result in Proposition 1 justifies our strategy to obtain a sufficient condition on the transmission rate to guarantee (1) is ISpS, which consists of finding conditions to achieve (9) for all reception times. Here we specify a quantization policy and determine the resulting estimation error as a function of the number of bits transmitted. This allows us to determine the packet size that ensures (14) (and consequently (9)) holds, thereby leading to a complete control design which ensures (1) is ISpS and rules out Zeno behavior. In turn, this also yields 
a sufficient condition on the information transmission rate. In our particular design the controller estimates $z\left(t_{c}\right)$ as

$$
\bar{z}\left(t_{c}\right)=\operatorname{sign}\left(z\left(t_{s}\right)\right) J e^{A\left(t_{c}-q\left(t_{s}\right)\right)},
$$

where $q\left(t_{s}\right)$ is an estimation of the triggering time $t_{s}$ constructed at the controller as described next. According to (7), at every triggering event, the sensor encodes $t_{s}$ and transmits a packet $p\left(t_{s}\right)$. The packet $p\left(t_{s}\right)$ consists of $g\left(t_{s}\right)$ bits of information and is generated according to the following quantization policy. The first bit $p\left(t_{s}\right)[1]$ denotes the sign of $z\left(t_{s}\right)$. As shown in Fig. 2, the reception time $t_{c}$ provides information

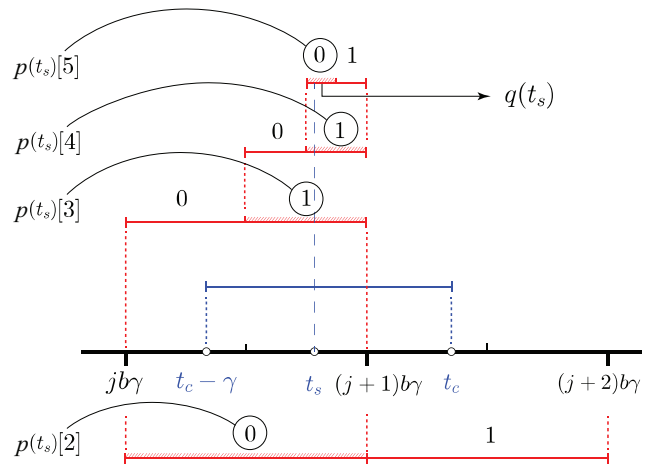

Fig. 2. The encoding-decoding algorithms in the proposed event-triggered control scheme. Here, $g\left(t_{s}\right)=5$ and $j$ is an even natural number. The packet $p\left(t_{s}\right)$ of length 5 can be generated and sent to the controller $\left(p\left(t_{s}\right)[1]\right.$ encodes the sign of $\left.z\left(t_{s}\right)\right)$. After reception and decoding, the controller chooses the center of the smallest sub-interval as its estimation of $t_{s}$, denoted by $q\left(t_{s}\right)$. to the controller that $t_{s}$ could fall anywhere between $t_{c}-\gamma$ and $t_{c}$. Let $b>1$. To determine the time interval of the triggering event, we break the positive time line into intervals of length $b \gamma$, cf. [48, Appendix C]. Consequently, $t_{s}$ falls into $[j b \gamma,(j+1) b \gamma]$ or $[(j+1) b \gamma,(j+2) b \gamma]$, with $j$ a natural number. We use the second bit of the packet to determine the correct interval of $t_{s}$. This bit is zero if the nearest integer less than or equal to the beginning number of the interval is an even number and is 1 otherwise. Mathematically, $p\left(t_{s}\right)[2]=\bmod \left(\left\lfloor\frac{t_{s}}{b \gamma}\right\rfloor, 2\right)$. For the remaining bits of the packet, the encoder breaks the interval containing $t_{s}$ into $2^{g\left(t_{s}\right)-2}$ equal sub-intervals. Once the packet is complete, it is transmitted to the controller, where it is decoded and the center point of the smallest sub-interval is selected as the best estimate of $t_{s}$. Thus,

$$
\left|t_{s}-q\left(t_{s}\right)\right| \leq b \gamma / 2^{g\left(t_{s}\right)-1} .
$$

Pseudo-code descriptions of the above encoding and decoding algorithms are provided in [48, Appendix D].

Remark 3: When the delay is sufficiently small, the timing information is substantial and the uncertainty about the value of the state at the controller is small. In this case, there is no need to resort to data payloads in the packet, as the plant can be stabilized using only timing information about the triggering events. In fact, the sensor simply transmits a fixed symbol from a unitary alphabet, reducing the communication channel to a telephone signaling channel [18] capable of stabilizing the system.

We have employed this quantization policy in our previous work [20] and analyzed its behavior in the case with no disturbances. Next, we extend our analysis to scenarios with both unknown delays and plant disturbances. As discussed in Remark 1, we start by showing that under the proposed encoding-decoding scheme, provided the sensor knows $\hat{x}(0)$ and has causal knowledge of the delay (i.e., the controller acknowledges the packet reception times), then Assumption 1 holds. The proof of the next result is in [48, Appendix B].

Proposition 2: Under the assumptions of Lemma 2, using the estimation (17) and the quantization policy described in Fig. 2, if the sensor has causal knowledge of delays (i.e., the controller acknowledges the packet reception times), then it can calculate $\left\{\hat{x}\left(t_{c}^{k+}\right)\right\}_{k \in \mathbb{N}}$.

2) Sufficient packet size: Our next result bounds the difference $\left|t_{s}-q\left(t_{s}\right)\right|$ between the triggering time and its quantized version so that (14) holds at all reception times.

Lemma 3: Consider the model with plant dynamics (1), estimator dynamics (4), triggering strategy (7), and jump strategy (8). Assume $|z(0)|=|x(0)-\hat{x}(0)|<J$. Using the estimation (17) and the quantization policy described in Fig. 2, if $\left|t_{s}-q\left(t_{s}\right)\right| \leq \frac{1}{A} \ln \left(1+\frac{\rho_{0}-\frac{M}{J A}\left(e^{A \gamma}-1\right)}{e^{A \gamma}}\right)$, then (14) holds for all reception times $\left\{t_{c}^{k}\right\}_{k \in \mathbb{N}}$ if $J>\frac{M}{A \rho_{0}}\left(e^{A \gamma}-1\right)$.

Proof: Using (11), (17), and the triangular inequality, we deduce $\left|z\left(t_{c}\right)-\bar{z}\left(t_{c}\right)\right| \leq J e^{A\left(t_{c}-t_{s}\right)}\left|\left(1-e^{A\left(t_{s}-q\left(t_{s}\right)\right)}\right)\right|+$ $\left|\int_{t_{s}}^{t_{c}} e^{A\left(t_{c}-\tau\right)} w(\tau) d \tau\right|$. By applying the bounds (3), (2), and (12) on first and second addend respectively it follows $\left|z\left(t_{c}\right)-\bar{z}\left(t_{c}\right)\right| \leq\left|J e^{A \gamma}\left(1-e^{A\left(t_{s}-q\left(t_{s}\right)\right)}\right)\right|+(M / A)\left(e^{A \gamma}-1\right)$. Therefore, ensuring (14) reduces to

$$
\left|1-e^{A\left(t_{s}-q\left(t_{s}\right)\right)}\right| \leq \eta,
$$

where $\eta=e^{-A \gamma}\left(\rho_{0}-\frac{M}{A J}\left(e^{A \gamma}-1\right)\right)$. Since $J>\frac{M}{A \rho_{0}}\left(e^{A \gamma}-1\right)$, we have $0 \leq \eta<1$. Consequently, using (19), we deduce $\ln (1-\eta) / A \leq t_{s}-q\left(t_{s}\right) \leq \ln (\eta+1) / A$. It follows that to satisfy (14) for all delay values, requiring $\left|t_{s}-q\left(t_{s}\right)\right| \leq$ $\min \{|\ln (1-\eta)| / A, \ln (1+\eta) / A\}$ suffices.

The next result provides a lower bound on the packet size so that (14) is ensured at all reception times.

Theorem 1: Consider the model with plant dynamics (1), estimator dynamics (4), triggering strategy (7), and jump strategy (8). Assume $|z(0)|=|x(0)-\hat{x}(0)|<J$. Then there exists a quantization policy that achieves (14) for all reception times $\left\{t_{c}^{k}\right\}_{k \in \mathbb{N}}$ with any packet size

$$
g\left(t_{s}^{k}\right) \geq \max \left\{0,1+\log \frac{A b \gamma}{\ln \left(1+\frac{\rho_{0}-(M /(J A))\left(e^{A \gamma}-1\right)}{e^{A \gamma}}\right)}\right\}
$$

where $b>1$ and $J>\frac{M}{A \rho_{0}}\left(e^{A \gamma}-1\right)$.

The proof is a direct consequence of (18) and Lemma 3. The combination of the upper bound (16) obtained for the triggering rate and Theorem 1 yields a sufficient bound on the information transmission rate. To sum it up, we conclude that there exists an information transmission rate

$R_{s} \leq$

$\frac{A}{\ln \left(\frac{J A+M}{\rho_{0} J A+M}\right)} \max \left\{0,1+\log \frac{A b \gamma}{\ln \left(1+\frac{\rho_{0}-(M /(J A))\left(e^{A \gamma}-1\right)}{e^{A \gamma}}\right)}\right\}$,

that is sufficient to ensure (14) and, as a consequence (9), for all reception times $\left\{t_{c}^{k}\right\}_{k \in \mathbb{N}}$. Therefore, from Proposition 1, the bound (21) is sufficient to ensure the plant (1) is ISpS.

Remark 4: The lower bound given on the packet size in (20) 
might not be a natural number or might even be zero. We use it to properly bound in (21) the information transmission rate $R_{s}$, which is a non-negative real number. For sufficiently small $\gamma$, if $g\left(t_{s}\right)=0$ is sufficient, the plant can be stabilized using only timing information and there is no need to put any data payload in the packet, cf. Remark 3. If we do not use fixed symbols as in telephone signaling channels [18], in practice, the packet size should be a natural number. Hence, we employ

$$
g\left(t_{s}\right)=\max \left\{1,\left\lceil 1+\log \frac{A b \gamma}{\ln \left(1+\frac{\rho_{0}-(M /(J A))\left(e^{A \gamma}-1\right)}{e^{A \gamma}}\right)}\right\rceil\right\},
$$

which is sufficient for stabilization (and is the one used in our simulations of Appendix A).

\section{B. Necessary information transmission rate}

Here, we present a necessary condition on the information transmission rate required by any control policy to render plant (1) ISpS under the class of event-triggering strategies described in Section III. In Section IV-A, to derive a sufficient bound that guarantees (1) is ISpS, our focus has been on identifying $a$ quantization policy that could handle any realization of initial condition, delay, and disturbance. Here, we focus on any quantization policy, for which we identify at least $a$ realization of initial condition, delay, and disturbance that requires the necessary bound on the information transmission rate.

Our strategy to provide a necessary condition for (1) to be ISpS is based on the following observation. Note that, if the property (9) was not satisfied at an arbitrary reception time $t_{c}^{k}$ (i.e., $z\left(t_{c}^{k}\right)>J$ ), and in addition either $w(t)>0$ or $w(t)<0$ for all $t \geq t_{c}^{k}$, then $t_{c}^{k}$ would be the last triggering time as (7) would never be satisfied again. Then, after $t_{c}^{k}$, the controller would need to estimate the inherently unstable plant in open loop. This would mean that there exists a realization of the initial condition, system disturbances, and delay for which the absolute value of the state estimation error grows exponentially with time. Thus, for any given control policy, there would exist a realization for which the absolute value of the state tends to infinity with time, and (1) is not ISpS.

As a consequence of this observation, our strategy to provide a necessary condition consists of identifying a necessary condition on the information transmission rate $R_{s}$ to have (9) at all reception times $\left\{t_{c}^{k}\right\}_{k \in \mathbb{N}}$. In turn, we do this by finding lower bounds on the packet size $g\left(t_{s}\right)$ and the triggering rate $R_{t r}$. We do this in two steps: first, we find a lower bound on the number of bits transmitted at each triggering event which holds irrespective of the triggering rate. Then, we find a lower bound on the triggering rate, and the combination leads us to the necessary condition on $R_{s}$.

1) Necessary packet size: We rely on (11) to define the uncertainty set of the sensor about the estimation error at the controller $z\left(t_{c}\right)$ given $t_{s}$ as follows

$$
\begin{array}{r}
\Omega\left(z\left(t_{c}\right) \mid t_{s}\right)=\left\{y: y= \pm J e^{A\left(t_{r}-t_{s}\right)}+\int_{t_{s}}^{t_{r}} e^{A\left(t_{r}-\tau\right)} w(\tau) d \tau\right. \\
\left.t_{r} \in\left[t_{s}, t_{s}+\gamma\right],|w(\tau)| \leq M \text { for } \tau \in\left[t_{s}, t_{r}\right]\right\} .
\end{array}
$$

Additionally, we define the uncertainty of the controller about $z\left(t_{c}\right)$ given $t_{c}$, as follows

$$
\begin{array}{r}
\Omega\left(z\left(t_{c}\right) \mid t_{c}\right)=\left\{y: y= \pm J e^{A\left(t_{c}-t_{r}\right)}+\int_{t_{r}}^{t_{c}} e^{A\left(t_{c}-\tau\right)} w(\tau) d \tau\right. \\
\left.t_{r} \in\left[t_{c}-\gamma, t_{c}\right],|w(\tau)| \leq M \text { for } \tau \in\left[t_{r}, t_{c}\right]\right\} .
\end{array}
$$

We next show the relationship between these uncertainty sets.

Lemma 4: Consider the model described in Section II, with plant dynamics (1), estimator dynamics (4), triggering strategy (7), and jump strategy (8). Moreover, assume $M \leq$ $A J$. Then $\Omega\left(z\left(t_{c}\right) \mid t_{s}\right)=\Omega\left(z\left(t_{c}\right) \mid t_{c}\right)$ and $m\left(\Omega\left(z\left(t_{c}\right) \mid t_{c}\right)\right)=$ $2(M / A+J)\left(e^{A \gamma}-1\right)$.

Proof: Due to symmetry, one can show that $\Omega\left(z\left(t_{c}\right) \mid t_{s}\right)$ is the same as $\Omega\left(z\left(t_{c}\right) \mid t_{c}\right)$. We characterize the set $\Omega\left(z\left(t_{c}\right) \mid t_{s}\right)$ as follows. We reason for the case when $z\left(t_{s}\right)=J$ (the argument for $z\left(t_{s}\right)=-J$ is analogous). Clearly, $z\left(t_{c}\right)$ takes its largest value when $t_{c}=t_{s}+\gamma$ and $w(\tau)=M$ for $\tau \in\left[t_{s}, t_{c}\right]$, which is equal to $z\left(t_{c}\right)=J e^{A \gamma}+(M / A)\left(e^{A \gamma}-1\right)$. Finding the smallest value of $z\left(t_{c}\right)$ is more challenging. When $t_{c}=t_{s}$,

$$
z\left(t_{c}\right)=J
$$

By setting $w(\tau)=-M$ for $\tau \in\left[t_{s}, t_{c}\right]$ and $t_{c}=t_{s}+\Delta$,

$$
z\left(t_{c}\right)=J e^{A \Delta}-(M / A)\left(e^{A \Delta}-1\right) .
$$

Taking the derivative of (24) with respect to $\Delta$ results in

$$
d z\left(t_{c}\right) / d \Delta=A J e^{A \Delta}-M e^{A \Delta}=e^{A \Delta}(A J-M) .
$$

If $M \leq A J$ and the derivative in (25) is non-negative, $z\left(t_{c}\right)$ in (24) would be a non-decreasing function of $\Delta$. Hence, the smallest value of $z\left(t_{c}\right)$ in (24) occurs for $\Delta=0$ which is equal to the value of $z\left(t_{c}\right)$ in (23). Hence, $\Omega\left(z\left(t_{c}\right) \mid t_{s}\right)=\left[J, J e^{A \gamma}+\right.$ $\left.(M / A)\left(e^{A \gamma}-1\right)\right]$, and the result follows.

Lemma 4 allows us to find a lower bound on the packet size $g\left(t_{s}\right)$, which is valid irrespective of the triggering rate.

Lemma 5: Under the assumptions of Lemma 4, if (9) holds for all reception times $\left\{t_{c}^{k}\right\}_{k \in \mathbb{N}}$, then the packet size at every triggering event must satisfy

$$
g\left(t_{s}^{k}\right) \geq \max \left\{0, \log \left((M /(A J)+1)\left(e^{A \gamma}-1\right)\right)\right\} .
$$

Proof: To ensure (9) for all reception times, we calculate a lower bound on the number of bits to be transmitted to ensure the sensor uncertainty set $\Omega\left(z\left(t_{c}\right) \mid t_{s}\right)$ is covered by quantization cells of measure $2 J$. Therefore, we have $g\left(t_{s}\right) \geq$ $\max \left\{0, \log \left(m\left(\Omega\left(z\left(t_{c}\right) \mid t_{s}\right)\right) / m(\mathcal{B}(J))\right)\right\}$, where $\mathcal{B}(J)$ is a ball centered at 0 of radius $J$, and we have incorporated the fact that the packet size $g\left(t_{s}\right)$ must be non-negative. From Lemma $4, \log \frac{m\left(\Omega\left(z\left(t_{c}\right) \mid t_{s}\right)\right)}{m(\mathcal{B}(J))} \geq \log \frac{(M / A+J)\left(e^{A \gamma}-1\right)}{J}$.

2) Lower bound on the triggering rate: Our next step is to determine a lower bound on the triggering rate.

Lemma 6: Under the assumptions of Lemma 4, for all the quantization policies which ensure (9) at all reception times $\left\{t_{c}^{k}\right\}_{k \in \mathbb{N}}$, if there exists a delay realization $\left\{\Delta_{k} \leq \alpha\right\}_{k \in \mathbb{N}}$, a disturbance realization, and an initial condition such that

$$
\left|z\left(t_{c}^{k+}\right)\right|=\left|z\left(t_{c}^{k}\right)-\bar{z}\left(t_{c}^{k}\right)\right| \geq \Upsilon
$$

for all $k \in \mathbb{N}$, then

$$
R_{t r} \geq A\left(\ln \left(e^{A \alpha}(J A+M) /(\Upsilon A+M)\right)\right)^{-1},
$$


for said delay realization, disturbance realization, and initial condition.

Proof: Using the definition of the triggering time (7), (27), $t_{c}^{k}=t_{s}^{k}+\Delta_{k}$, and (11), we have $\Upsilon e^{A\left(t_{s}^{k+1}-t_{s}^{k}-\Delta_{k}\right)}+$ $(M / A)\left(e^{A\left(t_{s}^{k+1}-t_{s}^{k}-\Delta_{k}\right)}-1\right) \leq J$, which is equivalent to

$$
e^{A\left(t_{s}^{k+1}-t_{s}^{k}\right)} \leq e^{A \Delta_{k}}(J A+M) /(\Upsilon A+M) .
$$

By hypothesis, (27) occurs for all $k \in \mathbb{N}$ when $\Delta_{k} \leq \alpha$. Hence, by (29), we upper bound the triggering intervals as

$$
\Delta_{k}^{\prime}=t_{s}^{k+1}-t_{s}^{k} \leq A^{-1} \ln \left(e^{A \alpha}(J A+M) /(\Upsilon A+M)\right) \text {.(30) }
$$

The result follows by substituting (30) into (15).

If we do not limit the collection of permissible quantization policies, a packet may carry an unbounded amount of information, which can bring the state estimation error arbitrarily close to zero at all reception times and for all delay and disturbance values. This would give rise to a conservative lower bound on the transmission rate. Specifically, using $\Delta_{k} \leq \gamma$, cf. (3), putting $\Upsilon=0$, and combining (28) and (26) we deduce there exists a delay realization, disturbance realization, and initial condition such that

$$
R_{s} \geq A \frac{\max \left\{0, \log \left(\left(\frac{M}{A J}+1\right)\left(e^{A \gamma}-1\right)\right)\right\}}{\ln \left(e^{A \gamma \frac{J A+M}{M}}\right)},
$$

is necessary for all quantization policies. To find a tighter necessary condition, we instead limit the collection of permissible quantization policies. Since ensuring (9) at each reception time is equivalent to dividing the uncertainty set at the controller $\Omega\left(z\left(t_{c}\right) \mid t_{c}\right)$ by quantization cells of measure at most $2 J$, our approach is to restrict the class of quantization policies to those that use the minimum possible number of bits to ensure (9).

Assumption 2: We assume at each triggering time the sensor transmits the smallest possible packet size to ensure (9) at each reception time for all initial conditions and all possible realizations of the delay and plant disturbance. Moreover, to simplify our analysis in the encoding-decoding scheme, we choose the center of each quantization cell as $\bar{z}\left(t_{c}\right)$.

Based on this assumption, the sensor brings the uncertainty about $z\left(t_{c}\right)$ at the controller down to a quantization cell of measure at most $2 J$, using the smallest possible packet size. The next result, whose proof is in [48, Appendix B], shows that, for this class of quantization policies, there exists a delay realization such that the sensor can only shrink the estimation error for the controller to at most half of $J$ dictated by (9).

Lemma 7: Let $\beta=\ln (1+2 A J /(A J+M)) / A \leq \gamma$. Under the assumptions of Lemma 4 , for all the quantization policies ensuring (9) at all reception times $\left\{t_{c}^{k}\right\}_{k \in \mathbb{N}}$ with Assumption 2 in place, there exists a delay realization $\left\{\Delta_{k} \leq\right.$ $\beta\}_{k \in \mathbb{N}}$, initial condition, and plant disturbance such that

$$
\left|z\left(t_{c}^{k+}\right)\right|=\left|z\left(t_{c}^{k}\right)-\bar{z}\left(t_{c}^{k}\right)\right| \geq J / 2 .
$$

Combining Lemmas 6 and 7, we deduce there exists a delay realization, disturbance realization, and initial condition so that

$$
R_{t r} \geq A\left(\ln \left(\left(1+\frac{2 A J}{A J+M}\right) \frac{J A+M}{0.5 J A+M}\right)\right)^{-1}
$$

is valid for all quantization policies that use the minimum required packet size according to Assumption 2.
Combining the bounds on the packet size (cf. Lemma 5) and on the triggering rate (cf. (32)), we obtain the following.

Theorem 2: Under the assumptions of Lemma 4, for all the quantization policies which ensure (9) at all reception times $\left\{t_{c}^{k}\right\}_{k \in \mathbb{N}}$ with Assumption 2 in place, there exists a delay realization $\left\{\Delta_{k} \leq \beta\right\}_{k \in \mathbb{N}}$, a disturbance realization, and an initial condition such that

$$
R_{s} \geq A \frac{\max \left\{0, \log \left((M /(A J)+1)\left(e^{A \gamma}-1\right)\right)\right\}}{\ln \left(\left(1+\frac{2 A J}{A J+M}\right) \frac{J A+M}{0.5 J A+M}\right)} .
$$

The bound (33) is tighter than the bound in (31). Fig. 3 compares our bounds on the sufficient (21) and necessary (33) information transmission rates for (1) to be ISpS. We attribute the gap between them to the fact that, while the necessary condition employs quantization policies with the minimum possible packet size according to Assumption 2, the encodingdecoding scheme in the sufficient design does not generally satisfy this assumption. The fact that we bound the triggering rate and the packet size independently in our analysis might further contribute to the gap. The key point that is evident from Fig. 3, is that for sufficiently small delay values the timing information is substantial, and the plant can be ISpS in the presence of bounded disturbances when the sensor transmits data payload at a smaller rate than the one prescribed by the data-rate theorem. As the delay increases, the timing information becomes less useful. Since the state estimation error is smaller than the triggering threshold at each reception time in our design, for larger values of delay, $R_{s}$ exceeds the access rate prescribed by the data-rate theorem.

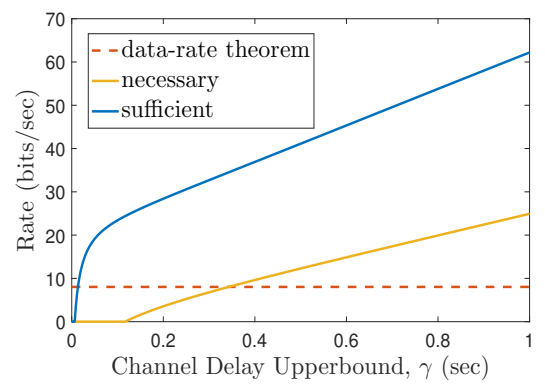

Fig. 3. Illustration of the sufficient (21) and necessary (33) transmission rates as functions of the delay upper bound $\gamma$. Here, $A=5.5651, \rho_{0}=0.1$, $b=1.0001, M=0.4$, and $J=\frac{M}{A \rho_{0}}\left(e^{A \gamma}-1\right)+0.1$. The rate dictated by the data-rate theorem is $R_{c} \geq A / \ln 2=8.02874$.

\section{EXTENSION TO COMPLEX LINEAR SYSTEMS}

In this section, we generalize our treatment to complex linear plants with disturbances. The results presented here can be readily applied to multivariate linear plants with disturbance and diagonalizable open loop-gain matrix (possibly, with complex eigenvalues). This corresponds to handling the $n$-dimensional real plant as $n$ scalar (and possibly complex) plants, and derive a sufficient condition for them. We consider a plant, sensor, communication channel and controller described by the continuous linear time-invariant system

$$
\dot{x}=A x(t)+B u(t)+w(t),
$$

where $x(t)$ and $u(t)$ belong to $\mathbb{C}$ for $t \in[0, \infty)$. Here $w(t) \in$ $\mathbb{C}$ represents a plant disturbance, which is upper bounded as 
$\|w(t)\| \leq M$, with $M \in \mathbb{R}_{\geq 0}$. Also, $A \in \mathbb{C}$ with $\operatorname{Re}(A) \geq 0$ (since we are only interested in unstable plants) and $B \in \mathbb{C}$ is nonzero. The model for the communication channel is the same as in Section II. To establish a baseline for comparison of the bounds on the information transmission rate, we start by stating a generalization of the classical data-rate theorem for the complex plant (34). The proof is in [48, Appendix B].

Theorem 3: Consider the model with plant dynamics (34). If $\|x(t)\|$ remains bounded as $t \rightarrow \infty$, then $R_{c} \geq 2 \operatorname{Re}(A) / \ln 2$.

\section{A. Event-triggered control for complex linear systems}

The state estimate $\hat{x}$ evolves according to the dynamics (4) along the inter-reception time intervals starting from $\hat{x}\left(t_{c}^{k+}\right)$ with initial condition $\hat{x}(0)$. We use the state estimation error defined as (5) with initial condition $z(0)=x(0)-\hat{x}(0)$. A triggering event happens at $t_{s}^{k+1}$ if

$$
\left\|z\left(t_{s}^{k+1}\right)\right\|=J
$$

provided $t_{c}^{k} \leq t_{s}^{k+1}$ for $k \in \mathbb{N}$ and $t_{s}^{1} \geq 0$, and the triggering radius $J \in \mathbb{R}$ is positive. At each triggering time, the packet $p\left(t_{s}\right)$ of size $g\left(t_{s}\right)$ is transmitted from the sensor to the controller. The packet $p\left(t_{s}\right)$ consists of a quantized version of the phase of $z\left(t_{s}\right)$, denoted $\phi_{q\left(z\left(t_{s}\right)\right)}$, and a quantized version of the triggering time $t_{s}$. By (35), we have $z\left(t_{s}\right)=J e^{i \phi_{z\left(t_{s}\right)}}$. We construct a quantized version, denoted $q\left(z\left(t_{s}\right)\right)$, of $z\left(t_{s}\right)$ at the controller as $q\left(z\left(t_{s}\right)\right)=J e^{i \phi_{q\left(z\left(t_{s}\right)\right)}}$. Additionally, using the bound (3) and the packet at the controller, the quantized version of $t_{s}$ is reconstructed and denoted by $q\left(t_{s}\right)$. Hence, at the controller, $z\left(t_{c}\right)$ is estimated as follows

$$
\bar{z}\left(t_{c}\right)=e^{A\left(t_{c}-q\left(t_{s}\right)\right)} q\left(z\left(t_{s}\right)\right) .
$$

We use the jump strategy (8) to update the value of $\hat{x}\left(t_{c}^{+}\right)$. Hence, $\left\|z\left(t_{c}^{+}\right)\right\|=\left\|z\left(t_{c}\right)-\bar{z}\left(t_{c}\right)\right\|$ holds. At the sensor, the packet size $g\left(t_{s}\right)$ is chosen to be large enough such that

$$
\left\|z\left(t_{c}^{+}\right)\right\|=\left\|z\left(t_{c}\right)-\bar{z}\left(t_{c}\right)\right\| \leq \rho_{0} J
$$

(where $0<\rho_{0}<1$ is a design parameter) is satisfied for all $t_{c} \in\left[t_{s}, t_{s}+\gamma\right]$. Fig. 4(a) shows a typical realization of $z(t)$ under the proposed event-triggered strategy before and after one event. The notion of ISpS is the same as in Definition 1 by replacing absolute value with complex absolute value.

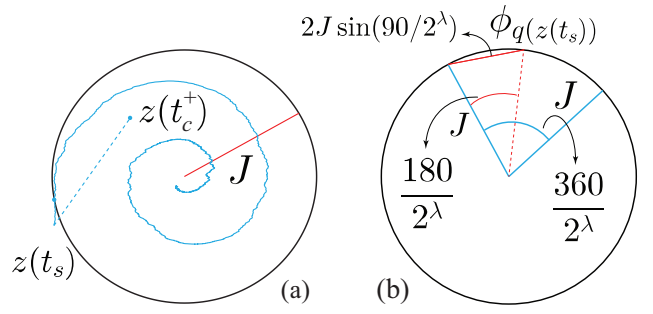

Fig. 4. (a) Evolution of the state estimation error (blue curve) before and after an event. The trajectory starts with an initial state inside a circle of radius $J$, and continues spiraling (due to the imaginary part of $A$ ) until it hits the threshold $J$. Then it jumps back inside the circle after the update according to (36) and jump strategy (8). During inter-reception time intervals, $\dot{z}(t)=A z(t)+w(t)$, and the observed overshoot beyond the circle is due to the delay in the communication channel. Here, $A=0.3+2 i, B=0.2$, $u(t)=-8 \hat{x}(t), M=0.2, \gamma=0.05 \mathrm{sec}, \rho_{0}=0.9$ and $J=0.0173$. (b) Estimation of the phase angle after event and transmission of $\lambda$ bits.
Remark 5: Similarly to Proposition 1, one can show that if (37) occurs at all reception times and $(A, B)$ is a stabilizable pair, then under the control rule $u(t)=-K \hat{x}(t)$, the plant (34) is ISpS, provided the real part of $A-B K$ is negative. As a consequence of this observation, our analysis focuses on ensuring (37) at each reception time. The lower bound on the inter-event time of Lemma 2 and the upper bound on the triggering rate (16) also holds replacing $A$ by $\operatorname{Re}(A)$.

\section{B. Sufficient information transmission rate}

We design a quantization policy that, using the eventtriggered controller of Section V-A, ensures the plant (34) is ISpS. We rely on this design to establish a sufficient bound on the information transmission rate.

1) Design of quantization policy: We devote the first $\lambda$ bits of the packet $p\left(t_{s}\right)$ for quantizing the phase of $z\left(t_{s}\right)$. The proposed encoding algorithm uniformly quantizes the circle into $2^{\lambda}$ pieces of $2 \pi / 2^{\lambda}$ radians. After reception, the decoder finds the correct phase quantization cell and selects its center point as $\phi_{q\left(z\left(t_{s}\right)\right)}$. By letting $\omega=\phi_{z\left(t_{s}\right)}-\phi_{q\left(z\left(t_{s}\right)\right)}$, as depicted in Fig. 4(b), geometrically we deduce $|\omega| \leq \pi / 2^{\lambda}$. Furthermore, we use the encoding scheme proposed in Fig. 2 to append a quantized version of triggering time $t_{s}$ of length $g\left(t_{s}\right)-\lambda$ to the packet $p\left(t_{s}\right)$. Hence, $p\left(t_{s}\right)[\lambda+1]=\bmod \left(\left\lfloor\frac{t_{s}}{b \gamma}\right\rfloor, 2\right)$. For the remaining bits of the packet, the encoder breaks the interval containing $t_{s}$ into $2^{g\left(t_{s}\right)-\lambda-1}$ equal sub-intervals. Once the packet is complete, it is transmitted to the controller, where it is decoded and the center point of the smallest sub-interval is selected as the best estimate of $t_{s}$. Therefore,

$$
\left|t_{s}-q\left(t_{s}\right)\right| \leq b \gamma / 2^{g\left(t_{s}\right)-\lambda} .
$$

Given $t_{s}^{k+1}$, one can identify $q\left(t_{s}^{k+1}\right)$ deterministically. Also, using the first $\lambda$ bits of the packet, the sensor can find the value of $\phi_{q\left(z\left(t_{s}\right)\right)}$. Similarly to Proposition 2, if the sensor has a causal knowledge of the delay in the channel, it can calculate $\hat{x}(t)$ for all time $t$.

2) Sufficient packet size: Here we show that with a sufficiently large packet size, we can achieve (37) at all reception times $\left\{t_{c}^{k}\right\}_{k \in \mathbb{N}}$ using the quantization policy designed in Section V-B1. The proof of the next result is in [48, Appendix B].

Theorem 4: Consider the model with plant dynamics (34), estimator dynamics (4), triggering strategy (35), and jump strategy (8). Assume $\|z(0)\|=\|x(0)-\hat{x}(0)\|<J$, then the quantization policy designed above achieves (37) for all reception times $\left\{t_{c}^{k}\right\}_{k \in \mathbb{N}}$ with any packet size lower bounded by

$g\left(t_{s}\right) \geq \bar{g} \triangleq$
$\max \left\{0, \lambda+\log \frac{\operatorname{Re}(A) b \gamma}{\ln \left(\frac{1+e^{-\operatorname{Re}(A) \gamma}\left(\rho_{0}-\frac{M}{\operatorname{Re}(A) J}\left(e^{\operatorname{Re}(A) \gamma}-1\right)\right)}{2 \sin \left(\pi / 2^{\lambda+1}\right)+1+\sqrt{2 \zeta}}\right)}\right\}$,

provided $\cos \left(\operatorname{Im}(A)\left(t_{s}-q\left(t_{s}\right)\right)\right)=1-\zeta, b>1$,

$\rho_{0} \geq$

$$
\begin{aligned}
& \frac{M}{\operatorname{Re}(A) J}\left(e^{\operatorname{Re}(A) \gamma}-1\right)+e^{\operatorname{Re}(A) \gamma}\left(2 \sin \left(\pi / 2^{\lambda+1}\right)+\sqrt{2 \zeta}\right), \\
& J \geq \frac{M}{\operatorname{Re}(A) \chi}\left(e^{\operatorname{Re}(A) \gamma}-1\right), \quad \sqrt{2 \zeta} e^{\operatorname{Re}(A) \gamma} \leq \chi^{\prime},
\end{aligned}
$$


$\lambda>\log \left(\pi / \arcsin \left(\frac{1-\chi-\chi^{\prime}}{2 e^{\operatorname{Re}(A) \gamma}}\right)\right)-1$,

where $0<\chi+\chi^{\prime}<1$.

Combining the bound on the triggering rate from Remark 5 with Theorem 4, it follows that there exists an information transmission rate with

$$
R_{s} \leq \operatorname{Re}(A) \bar{g} / \ln \left(\frac{J \operatorname{Re}(A)+M}{\rho_{0} J \operatorname{Re}(A)+M}\right),
$$

that achieves (37) for all reception times $\left\{t_{c}^{k}\right\}_{k \in \mathbb{N}}$, and is sufficient to ensure (34) is ISpS. Fig. 5 plots the rate in (40) as a function of the delay upper bound $\gamma$. For small values of the delay, the sufficient information transmission rate is smaller than the rate required by the data-rate result in Theorem 3 .

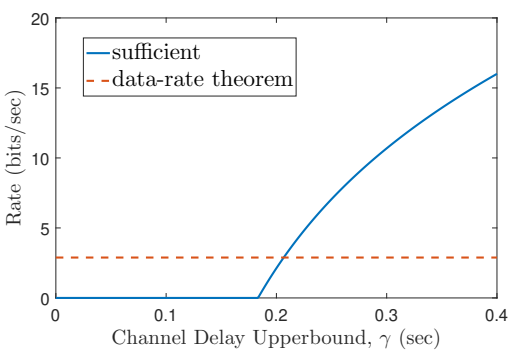

Fig. 5. Sufficient information transmission rate (40) as a function of channel delay upper bound $\gamma$. Here $A=1+i, B=0.5, M=0.1$, $\rho_{0}=0.9$ and $b=1.0001$. Also $\lambda=\log \left(\pi / 2 \arcsin \left(\frac{7}{8}\right) e^{\operatorname{Re}(A) \gamma}\right)$ and $J=\frac{8 M}{\operatorname{Re}(A)}\left(e^{\operatorname{Re}(A) \gamma}-1\right)+0.002$. The rate dictated by the data-rate theorem (cf. Theorem 3) is $2 \operatorname{Re}(A) / \ln 2=2.885$.

Remark 6: Following the discussion of Remarks 3 and 4, when $\bar{g}=0$ in (38), there is no need for any data payload, and (34) can be stabilized using only timing information.

Remark 7: Depending on whether the system is real or complex, the corresponding triggering criterion is based on the real or complex absolute value, resp., cf. (7) and (35). The controller needs to approximate the phase at which the state estimation error $z\left(t_{s}\right)$ hits the triggering radius. The real case is a special case, since the phase of $z\left(t_{s}\right)$ is then either 0 or $\pi$. I this case in the sufficient design only the first bits of the packet $p\left(t_{s}\right)$ denote the sign of $z\left(t_{s}\right)$. In the complex case, we use the first $\lambda$ bits of the packet $p\left(t_{s}\right)$ for quantizing the phase of $z\left(t_{s}\right)$. By putting $A=\operatorname{Re}(A), \lambda=1$, and $\operatorname{Im}(A)=0$ (or $\zeta=0$ ), our sufficient condition for complex systems (40), becomes (21) except a factor $1+\sqrt{2}$, which makes (40) larger than (21). The reason for the difference is the obtained upper bound in this case for the estimation error of the phase of $z\left(t_{s}\right)$ (see [48, Eq. (50) in Appendix B]). In the real case, the controller deduces $z\left(t_{s}\right)=J$ or $z\left(t_{s}\right)=-J$, and the estimation error of the phase of $z\left(t_{s}\right)$ is zero.

\section{Vi. Conclusions}

We have presented an event-triggered control scheme for the stabilization of noisy, scalar real and complex, continuous, linear time-invariant systems over a communication channel subject to random bounded delay. We have developed an algorithm for encoding-decoding the quantized version of the estimated state, leading to the characterization of a sufficient transmission rate for stabilizing these systems. We also identified a necessary condition on the transmission rate for real systems. Future work will study the identification of necessary conditions on the transmission rate in complex systems, develop event-triggered designs for vector systems with real and complex eigenvalues, and the investigation of optimal values for the design parameters that balance the tradeoffs between transmission rate and control performance.

\section{REFERENCES}

[1] J. P. Hespanha, P. Naghshtabrizi, and Y. Xu, "A survey of recent results in networked control systems," Proceedings of the IEEE, vol. 95, no. 1, pp. 138-162, 2007.

[2] G. N. Nair, F. Fagnani, S. Zampieri, and R. J. Evans, "Feedback control under data rate constraints: An overview," Proceedings of the IEEE, vol. 95 , no. 1, pp. 108-137, 2007.

[3] S. Fang, J. Chen, and I. Hideaki, Towards integrating control and information theories. Springer, 2017.

[4] S. Tatikonda and S. Mitter, "Control under communication constraints," IEEE Tran. Auto. Cont., vol. 49, no. 7, pp. 1056-1068, 2004.

[5] G. N. Nair and R. J. Evans, "Stabilizability of stochastic linear systems with finite feedback data rates," SIAM Journal on Cont. and Optimization, vol. 43, no. 2, pp. 413-436, 2004.

[6] D. Liberzon, "On stabilization of linear systems with limited information," IEEE Tran. Auto. Cont., vol. 48, no. 2, pp. 304-307, 2003.

[7] A. Sahai and S. Mitter, "The necessity and sufficiency of anytime capacity for stabilization of a linear system over a noisy communication link. Part I: Scalar systems," IEEE Tran. on Info. Theory, vol. 52, no. 8 , pp. 3369-3395, 2006.

[8] A. S. Matveev and A. V. Savkin, Estimation and control over communication networks. Springer Science \& Business Media, 2009.

[9] S. Yüksel and T. Başar, Stochastic Networked Control Systems: Stabilization and Optimization under Information Constraints. Springer Sci. \& Bus. Med., 2013.

[10] G. Como, F. Fagnani, and S. Zampieri, "Anytime reliable transmission of real-valued information through digital noisy channels," SIAM J. Control Optimiz., vol. 48, no. 6, pp. 3903-3924, 2010.

[11] G. Yang and D. Liberzon, "Feedback stabilization of switched linear systems with unknown disturbances under data-rate constraints," IEEE Tran. Auto. Cont., vol. 63, no. 7, pp. 2107-2122, 2017.

[12] N. C. Martins, M. A. Dahleh, and N. Elia, "Feedback stabilization of uncertain systems in the presence of a direct link," IEEE Tran. Auto. Cont., vol. 51, no. 3, pp. 438-447, 2006.

[13] C. De Persis, "n-bit stabilization of n-dimensional nonlinear systems in feedforward form," IEEE Tran. Auto. Cont., vol. 50, no. 3, pp. 299-311, 2005.

[14] G. N. Nair, R. J. Evans, I. M. Mareels, and W. Moran, "Topological feedback entropy and nonlinear stabilization," IEEE Tran. Auto. Cont., vol. 49, no. 9, pp. 1585-1597, 2004.

[15] J. Ding, Y. Peres, G. Ranade, and A. Zhai, "When multiplicative noise stymies control," Ann. Appl. Probab., vol. 29, no. 4, pp. 1963-1992, 2019.

[16] V. Kostina and B. Hassibi, "Rate-cost tradeoffs in control," IEEE Tran. Auto. Cont., vol. 64, no. 11, pp. 4525-4540, 2019.

[17] A. Khina, E. R. Garding, G. M. Pettersson, V. Kostina, and B. Hassibi, "Control over Gaussian channels with and without source-channel separation," IEEE Tran. Auto. Cont., vol. 64, no. 9, pp. 3690-3705, 2019.

[18] V. Anantharam and S. Verdú, "Bits through queues," IEEE Tran. on Info. Theory, vol. 42, no. 1, pp. 4-18, 1996.

[19] W. P. M. H. Heemels, K. H. Johansson, and P. Tabuada, "An introduction to event-triggered and self-triggered control," in IEEE Conf. Decis. and Cont. (CDC), 2012, pp. 3270-3285.

[20] M. J. Khojasteh, P. Tallapragada, J. Cortés, and M. Franceschetti, "The value of timing information in event-triggered control," IEEE Tran. Auto. Cont., vol. 65, no. 3, pp. 925-940, 2020.

[21] A. Tanwani and A. Teel, "Stabilization with event-driven controllers over a digital communication channel with random transmissions," in IEEE Conf. Decis. and Cont. (CDC), 2017, pp. 6063-6068.

[22] B. Demirel, V. Gupta, D. E. Quevedo, and M. Johansson, "On the tradeoff between communication and control cost in event-triggered dead-beat control," IEEE Tran. Auto. Cont., vol. 62, no. 6, pp. 2973-2980, June 2017.

[23] L. Li, X. Wang, and M. Lemmon, "Stabilizing bit-rate of disturbed event triggered control systems," IFAC Proc. Vol., vol. 45, no. 9, pp. 70-75, 2012. 
[24] W. P. M. H. Heemels, M. Donkers, and A. R. Teel, "Periodic eventtriggered control for linear systems," IEEE Tran. Auto. Cont., vol. 58, no. 4, pp. 847-861, 2012.

[25] A. Tanwani, C. Prieur, and M. Fiacchini, "Observer-based feedback stabilization of linear systems with event-triggered sampling and dynamic quantization," Sys. \& Cont. Letters, vol. 94, pp. 46-56, 2016.

[26] N. Guo and V. Kostina, "Optimal causal rate-constrained sampling of the wiener process," in 57th Ann. Allerton Conf. on Comm., Cont., and Comp. (Allerton). IEEE, 2019, pp. 1090-1097.

[27] J. Pearson, J. P. Hespanha, and D. Liberzon, "Control with minimal costper-symbol encoding and quasi-optimality of event-based encoders," IEEE Tran. Auto. Cont., vol. 62, no. 5, pp. 2286-2301, 2017.

[28] E. Kofman and J. H. Braslavsky, "Level crossing sampling in feedback stabilization under data-rate constraints," in IEEE Conf. Decis. and Cont. (CDC), 2006, pp. 4423-4428.

[29] Q. Ling, "Bit rate conditions to stabilize a continuous-time scalar linear system based on event triggering," IEEE Tran. Auto. Cont., vol. 62, no. 8, pp. 4093-4100, 2017.

[30] — "Bit-rate conditions to stabilize a continuous-time linear system with feedback dropouts," IEEE Tran. Auto. Cont., vol. 63, no. 7, pp. 2176-2183, July 2018.

[31] S. Linsenmayer, R. Blind, and F. Allgöwer, "Delay-dependent data rate bounds for containability of scalar systems," IFAC-PapersOnLine, vol. 50, no. 1, pp. 7875-7880, 2017.

[32] A. K. Dhulipala, C. Fragouli, and A. Orlitsky, "Silence-based communication," IEEE Tran. on Info. Theory, vol. 56, no. 1, pp. 350-366, 2009.

[33] H. Yildiz, Y. Su, A. Khina, and B. Hassibi, "Event-triggered stochastic control via constrained quantization," in Data Comp. Conf. IEEE, 2019, pp. 612-612.

[34] Z.-P. Jiang, A. R. Teel, and L. Praly, "Small-gain theorem for ISS systems and applications," Mathematics of Control, Signals and Systems, vol. 7, no. 2, pp. 95-120, 1994.

[35] Y. Sharon and D. Liberzon, "Input to state stabilizing controller for systems with coarse quantization," IEEE Tran. Auto. Cont., vol. 57, no. 4, pp. 830-844, 2012.

[36] M. J. Khojasteh, M. Hedayatpour, J. Cortés, and M. Franceschetti, "Event-triggered stabilization of disturbed linear systems over digital channels," in Annual Conference on Info. Sciences and Sys. (CISS), March 2018

[37] _ - "Event-triggering stabilization of complex linear systems with disturbances over digital channels," in IEEE Conf. Decis. and Cont. (CDC), 2018, pp. 152-157.

[38] R. Siegwart, I. R. Nourbakhsh, and D. Scaramuzza, Introduction to autonomous mobile robots. MIT press, 2011.

[39] K. You and L. Xie, "Minimum data rate for mean square stabilization of discrete LTI systems over lossy channels," IEEE Tran. Auto. Cont., vol. 55, no. 10, pp. 2373-2378, 2010.

[40] S. Yuksel and S. P. Meyn, "Random-time, state-dependent stochastic drift for Markov chains and application to stochastic stabilization over erasure channels," IEEE Tran. on Auto. Cont., vol. 58, no. 1, pp. 47-59, 2012.

[41] V. Gupta, A. F. Dana, J. P. Hespanha, R. M. Murray, and B. Hassibi, "Data transmission over networks for estimation and control," IEEE Tran. on Auto. Cont., vol. 54, no. 8, pp. 1807-1819, 2009.

[42] S. Tatikonda and S. Mitter, "Control over noisy channels," IEEE Tran. Auto. Cont., vol. 49, no. 7, pp. 1196-1201, 2004.

[43] G. N. Nair, "A non-stochastic information theory for communication and state estimation," IEEE Tran. Auto. Cont., vol. 58, pp. 1497-1510, 2013.

[44] H. Shingin and Y. Ohta, "Disturbance rejection with information constraints: Performance limitations of a scalar system for bounded and Gaussian disturbances," Automatica, vol. 48, no. 6, pp. 1111-1116, 2012.

[45] J. P. Hespanha, A. Ortega, and L. Vasudevan, "Towards the control of linear systems with minimum bit-rate," in Proc. 15th Int. Symp. on Mathematical Theory of Networks and Systems (MTNS), 2002.

[46] J. Peralez, V. Andrieu, M. Nadri, and U. Serres, "Event-triggered output feedback stabilization via dynamic high-gain scaling," IEEE Tran. Auto. Cont., vol. 63, no. 8, pp. 2537-2549, 2018.

[47] R. Postoyan, A. Anta, D. Nešić, and P. Tabuada, "A unifying Lyapunovbased framework for the event-triggered control of nonlinear systems," in IEEE Conf. Decis. and Cont. and Eur. Cont. Conf., 2011, pp. 25592564.

[48] M. J. Khojasteh, M. Hedayatpour, J. Cortés, and M. Franceschetti, "Exploiting timing information in event-triggered stabilization of linear systems with disturbances," arXiv preprint: 1805.01969, 2018.

[49] M. J. Khojasteh, M. Hedayatpour, and M. Franceschetti, "Theory and implementation of event-triggered stabilization over digital channels," in IEEE Conf. Decis. and Cont. (CDC), Nice, France, 2019, pp. 41834188.

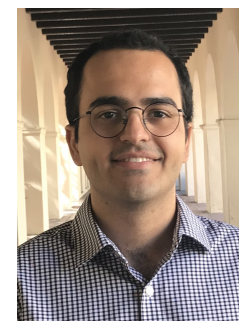

Mohammad Javad Khojasteh (S'14) did his undergraduate studies at Sharif University of Technology from which he received double-major B.Sc. degrees in Electrical Engineering and in Pure Mathematics, in 2015. He received the M.Sc. and Ph.D. degrees in Electrical and Computer Engineering from University of California San Diego (UCSD), La Jolla, CA, in 2017, and 2019, respectively. Currently, he is a Postdoctoral Scholar in the Center for Autonomous Systems and Technologies (CAST) at California Institute of Technology, Pasadena, CA, and a visitor at NASA's Jet Propulsion Laboratory (JPL).

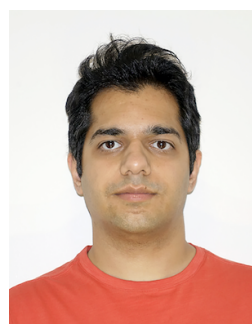

Mojtaba Hedayatpour received his bachelor's degree in aerospace engineering from Sharif University of Technology, Iran and his master's degree in industrial systems engineering from University of Regina, Canada. He has worked as Autonomous Systems Engineer at a startup company called Dot Technology Corporation in Canada where he developed guidance, navigation and control solutions for autonomous farming vehicles. He is currently the head of Artificial Intelligence team at the same company in Edmonton, Canada, where together with his team they develop autonomous solutions for farming applications.

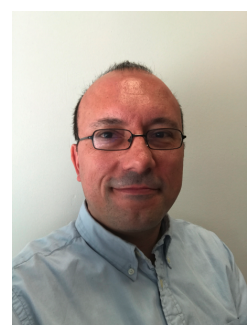

Jorge Cortés (M'02-SM'06-F'14) received the Licenciatura degree in mathematics from Universidad de Zaragoza, Zaragoza, Spain, in 1997, and the $\mathrm{Ph} . \mathrm{D}$. degree in engineering mathematics from Universidad Carlos III de Madrid, Madrid, Spain, in 2001. He held postdoctoral positions with the University of Twente, Twente, The Netherlands, and the University of Illinois at Urbana-Champaign, Urbana, IL, USA. He was an Assistant Professor with the Department of Applied Mathematics and Statistics, University of California, Santa Cruz, CA, USA, from 2004 to 2007. He is currently a Professor in the Department of Mechanical and Aerospace Engineering, University of California, San Diego, CA, USA. He is the author of Geometric, Control and Numerical Aspects of Nonholonomic Systems (Springer-Verlag, 2002) and co-author (together with F. Bullo and S. Martínez) of Distributed Control of Robotic Networks (Princeton University Press, 2009). He is a Fellow of IEEE and SIAM. At the IEEE Control Systems Society, he has been a Distinguished Lecturer (2010-2014), and is currently its Director of Operations and an elected member (2018-2020) of its Board of Governors. His current research interests include distributed control and optimization, network science, opportunistic state-triggered control and coordination, reasoning under uncertainty, and distributed decision making in power networks, robotics, and transportation. 


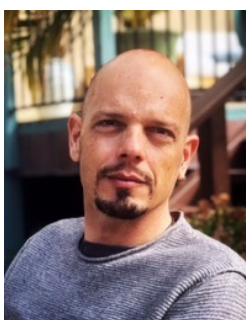

Massimo Franceschetti received the Laurea degree (with highest honors) in computer engineering from the University Federico II, Naples, Italy, in 1997, the M.S. and Ph.D. degrees in electrical engineering from the California Institute of Technology, in 1999, and 2003, respectively. He is Professor of Electrical and Computer Engineering at the University of California at San Diego (UCSD). Before joining UCSD, he was a postdoctoral scholar at the University of California at Berkeley for two years. He has held visiting positions at the Vrije Universiteit Amsterdam, the École Polytechnique Fédérale de Lausanne, and the University of Trento. His research interests are in physical and information-based foundations of communication and control systems. He is co-author of the book "Random Networks for Communication" and author of "Wave Theory of Information," both published by Cambridge University Press $(2007,2018)$. He was awarded the C. H. Wilts Prize in 2003 for best doctoral thesis in electrical engineering at Caltech; the S.A. Schelkunoff Award in 2005 for best paper in the IEEE Transactions on Antennas and Propagation, a National Science Foundation (NSF) CAREER award in 2006, an Office of Naval Research (ONR) Young Investigator Award in 2007, the IEEE Communications Society Best Tutorial Paper Award in 2010, and the IEEE Control theory society Ruberti young researcher award in 2012. He has been elected fellow of the IEEE in 2018 and became a Guggenheim fellow for the natural sciences, engineering, in 2019.

\section{APPENDIX A}

SimUlations

This section presents simulation results validating the proposed event-triggered control scheme for real-valued plants (the interested reader can find simulations for a complexvalued plant in [37]). While our analysis is for continuoustime plants, we perform the simulations in discrete time with a small sampling time $\delta^{\prime}>0$. Thus, the minimum upper bound for the communication network delay is equal to two sampling times in the digital environment (this is because a delay of at most one sampling time might occur from the time that triggering occurs to the time that the sensor took a sample from the plant state and another delay of at most one sampling time might occur from the time that the packet is received to the time the control input is applied to the plant). We consider a linearized version of the two-dimensional problem of balancing an inverted pendulum mounted on a cart, where the motion of the pendulum is constrained in a plane and its position can be measured by an angle $\theta$. The inverted pendulum has mass $m_{1}$, length $l$, and moment of inertia $I$. Also, the pendulum is mounted on top of a cart of mass $m_{2}$, constrained to move in $y$ direction. The nonlinear equations governing the motion of the cart and pendulum are $\left(m_{1}+m_{2}\right) \ddot{y}+\nu \dot{y}+m_{1} l \ddot{\theta} \cos \theta-m_{1} l \dot{\theta}^{2} \sin \theta=F$ and $\left(I+m_{1} l^{2}\right) \ddot{\theta}+m_{1} g_{0} l \sin \theta=-m_{1} l \ddot{y} \cos \theta$, where $\nu$ is the damping coefficient between the pendulum and the cart and $g_{0}$ is the gravitational acceleration. We define $\theta=\pi$ as the equilibrium position of the pendulum and $\phi$ as small deviations from $\theta$. We derive the linearized equations of motion using small angle approximation, noting that this linearization is only valid for sufficiently small values of the delay upper bound $\gamma$. Define the state variable $s=[y, \dot{y}, \phi, \dot{\phi}]^{T}$, where $y$ and $\dot{y}$ are the position and velocity of the cart respectively. Assuming $m_{1}=0.2 \mathrm{~kg}, m_{2}=0.5 \mathrm{~kg}, \nu=0.1 \mathrm{~N} / \mathrm{m} / \mathrm{s}, l=0.3$ $\mathrm{m}, I=0.006 \mathrm{~kg} / \mathrm{m}^{2}$, one can write the evolution of $s$ as

$$
\dot{s}=A s(t)+B u(t)+w(t),
$$

where

$$
A=\left[\begin{array}{cccc}
0 & 1 & 0 & 0 \\
0 & -0.1818 & 2.6730 & 0 \\
0 & 0 & 0 & 1 \\
0 & -0.4545 & 31.1800 & 0
\end{array}\right], B=\left[\begin{array}{c}
0 \\
1.8180 \\
0 \\
4.5450
\end{array}\right]
$$

In addition, we add the plant noise $w(t) \in \mathbb{R}^{4}$ to the linearized plant model, and we assume that all of its elements are upper bounded by $M$. A simple feedback control law can be derived for (41) as $u=-K s$, where $K=[-1.00-2.0420 .36$ 3.93], is chosen such that $A-B K$ is Hurwitz.

The eigenvalues of the open-loop gain of the plant $A$ are $e=\left[\begin{array}{lll}0 & -5.6041-0.14285 .5651\end{array}\right]$. Thus, the open-loop gain of the plant $A$ is diagonalizable (all eigenvalues of $A$ are distinct). Using the eigenvector matrix $P$, we diagonalize the plant to obtain

$$
\dot{\tilde{s}}=\tilde{A} \tilde{s}(t)+\tilde{B} \tilde{u}(t)+\tilde{w}(t),
$$

where

$$
\tilde{A}=\left[\begin{array}{cccc}
0 & 0 & 0 & 0 \\
0 & -5.6041 & 0 & 0 \\
0 & 0 & -0.1428 & 0 \\
0 & 0 & 0 & 5.5651
\end{array}\right], \quad \tilde{B}=\left[\begin{array}{c}
10.0000 \\
-2.3865 \\
10.0979 \\
2.2513
\end{array}\right] \text {, }
$$

where $\tilde{s}(t)=P^{-1} s(t)$ and $\tilde{w}(t)=P^{-1} w(t)$. Also, $\tilde{u}(t)=$ $-\tilde{K} \tilde{s}(t)$ where $\tilde{K}=K P$.

For the first three coordinates of the diagonalized plant in (42) the state estimation $\hat{s}$ at the controller simply constructs as $\dot{\hat{s}}_{i}=\tilde{A}_{i} \hat{s}(t)+\tilde{B}_{i} \tilde{u}(t)$, starting from $\hat{s}_{i}(0)$ for $i \in\{1,2,3\}$, where $\tilde{A}_{i}$ and $\tilde{B}_{i}$ denote the $i^{\text {th }}$ row of $\tilde{A}$ and $\tilde{B}$. Since the first three eigenvalues of $A$ are non-positive, they are inherently stable. Thus, by the data theorem [35] there is no need to use the communication network for them, and since $\tilde{A}-\tilde{B} \tilde{K}$ is Hurwitz, $\tilde{u}(t)=-\tilde{K} \tilde{s}(t)$ renders them ISS with respect to system disturbances. Now we apply Theorem 1 to the fourth mode of the plant, which is unstable, to make the whole plant ISpS. In fact, we use the packet size given in (22) for the simulations. Using the problem formulation in Section II, the estimated state for the unstable mode $\hat{s}_{4}$ evolves during the inter-reception times as

$$
\dot{\hat{s}}_{4}(t)=5.5651 \hat{s}_{4}(t)+2.2513 \tilde{u}(t), \quad t \in\left(t_{c}^{k}, t_{c}^{k+1}\right),
$$

starting from $\hat{s}_{4}\left(t_{c}^{k+}\right)$ and $\hat{s}_{4}(0)$. Also, a triggering occurs when $\left|\tilde{z}_{4}(t)\right|=\left|\tilde{s}_{4}(t)-\hat{s}_{4}(t)\right|=J$, where $\left|\tilde{z}_{4}(t)\right|$ is the estate estimation error for the unstable mode, and assuming the previous packet is already delivered to the controller. In the simulation environment, since the sampling time is small, a triggering happens as soon as $\left|\tilde{z}_{4}(t)\right|$ is equal or greater than $J$ and the previous packet has been received by the controller. Let $\lambda_{4}=5.5651$ be the eigenvalue corresponding to the unstable mode. By Theorem 1, we choose $J=\left(M /\left(\lambda_{4} \rho_{0}\right)\right)\left(e^{\lambda_{4} \gamma}-1\right)+0.005$, and the size of the packet for all $t_{s}$ to be (22), where $b=1.0001$ and $\rho_{0}=0.9$.

Fig. 6(a) shows the triggering threshold for $\tilde{s}_{4}$ in (42) and the absolute value of the state estimation error for the unstable coordinate, that is, $\left|\tilde{z}_{4}(t)\right|=\left|\tilde{s}_{4}(t)-\hat{s}_{4}(t)\right|$. As soon as the absolute value of this error is equal or greater than the triggering threshold, the sensor transmits a packet, and the jumping strategy adjusts $\hat{s}_{4}$ at the reception time to ensure 


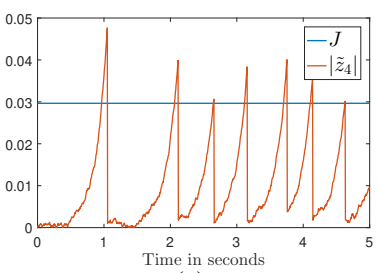

(a)

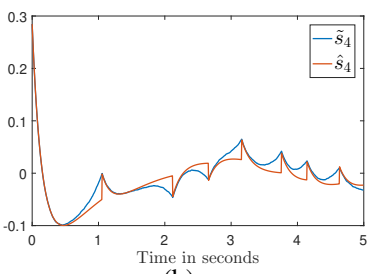

(b)

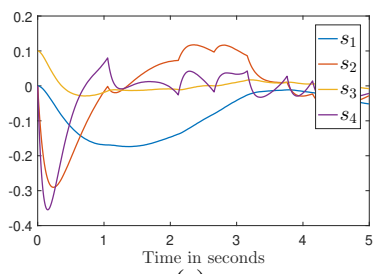

(c)

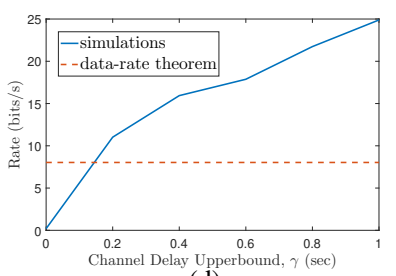

(d)

Fig. 6. Simulation results for the linearized inverted pendulum on a cart example. (a) shows the evolution of the absolute value of the state estimation error (a) for the unstable mode of the plant in (42). (b) shows the evolution of the unstable state in (42) and its estimate in (43). (c) shows the evolution of all the states in (41). (d) shows the information transmission rate in the simulation as compared to the data-rate theorem. Note that the rate does not start at $\gamma=0$ because the minimum channel delay upper bound is equal to two sampling times ( 0.005 seconds in this example). The simulation parameters are $\tilde{s}(0)=P^{-1}[0,0,0,0.1001]^{T}, \hat{s}(0)=P^{-1}[0,0,0,0.10]^{T}$, simulation time $T=5$ seconds, and sampling time $\delta^{\prime}=0.005$ seconds, For $(\mathrm{a})-(\mathrm{c}), \gamma=0.1$ sec, $g\left(t_{s}\right)=4$ bits, $M=0.05$, and in (d) $g\left(t_{s}\right)$ is calculated using (22) with $M=0.2$.

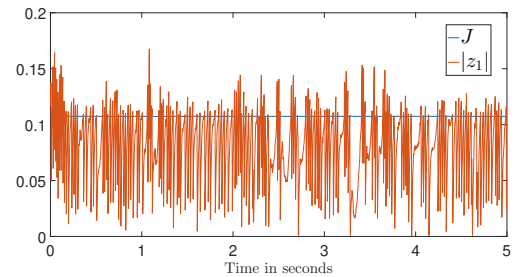

(a)

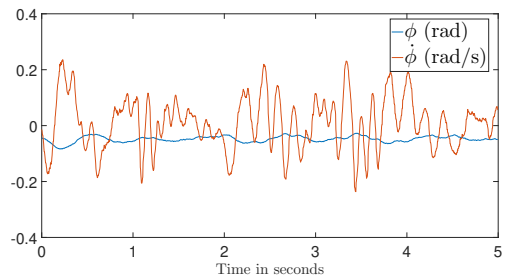

(b)

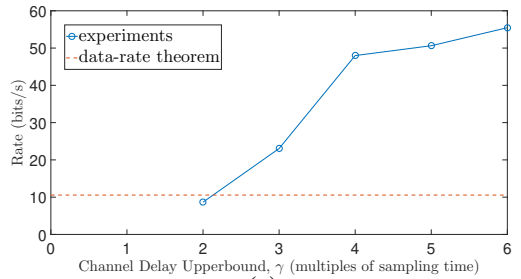

(c)

Fig. 7. Experimental results for controlling an inverted pendulum with the proposed event-triggered control strategy. (a) shows the triggering threshold $J$ and the estimation error in the pendulum's angular position $z_{1}=\phi-\hat{\phi}$, where $\phi$ is the sensor measurement and $\phi$ is the estimate of the angular position. (b) shows the actual angular position and velocity of the pendulum, with the former staying close to zero degrees (the desired upright position). In (a) and (b) the delay upper bound is set to five sampling times of the system (which is equal to 0.015 seconds) and the packet size is found to be 7 bits. (c) shows the information transmission rate in the experiments compared with the entropy rate of the system. The rate calculated from the experiments does not start at zero because the minimum channel delay upper bound is equal to two sampling times ( 0.006 seconds). The entropy rate of the system is 10.56 bits/sec, while the minimum transmission rate for delay bound equal to two sampling times is $8.66 \mathrm{bits} / \mathrm{sec}$.

the plant is ISpS. Note that the amount this error exceeds the triggering threshold depends on the random communication network delay upper bounded by $\gamma$. Fig. 6(b) presents the evolution of the unstable state in (42) and its estimation in (43). Fig. 6(c) shows the evolution of all the actual states of the linearized plant (41). Finally, Fig. 6(d) presents the simulation of information transmission rate versus the delay upper bound $\gamma$ in the communication network for stabilizing the linearized model of the inverted pendulum. For small $\gamma$, the plant is ISpS with an information transmission rate smaller than the one prescribed by the data-rate theorem.

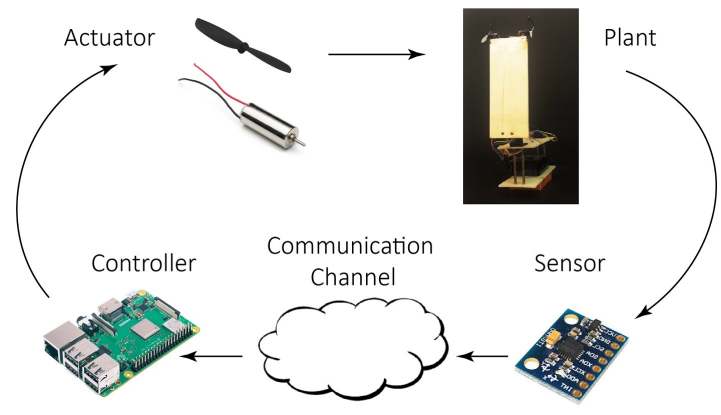

Fig. 8. Architecture and components of the prototype.

Remark 8: For further validation, we have also experimentally implemented the proposed event-triggered control strategy on an inverted pendulum controlled by two propellers as shown in Fig. 8. The robot used for the experiments is built using off-the-shelf components. Specifically, the frame is built with plywood sheets, we employ an InvenSense MPU6050 MEMS sensor (which consists of a 3-axis accelerometer and a 3-axis gyroscope), using a complementary filter to estimate the pendulum's angle and angular velocity, and we have a Raspberry Pi Model 3 acting as the computation unit as well as the controller. Finally, two small DC motors equipped with two identical propellers are used as actuators. Fig. 7(a) shows the evolution of the pendulum angle estimation error $z_{1}$ in time and Fig. 7(b) shows the angular position and velocity of the pendulum, where zero angle represents the upright position of the pendulum. We also ran a second set of experiments and calculated the information transmission rates using (22) as a function of the delay upper bound, cf. Fig. 7(c). The reason for the larger number of jumps in the experiments compared to the simulation is due to the additional uncertainty introduced by the nonlinear behavior of the system. Nevertheless, the same qualitative phase transition behavior is observed in Fig. 6(d) and Fig. 7(c). The interested reader is referred to [49] for further details of these experiments and validation ${ }^{3}$.

Remark 9: Several plots and discussions that illustrate the dependency of our sufficient (21) and necessary (33) rates on the plant disturbances $M$ and the design parameter $J$, along with plots and discussions that illustrate the effect of design parameters $b$ and $\rho_{0}$ on the sufficient rate (21) are available in $[48$, Appendix C].

\footnotetext{
${ }^{3}$ The code can also be found at https://github.com/mkhojas/Event-Triggered-Firmware.
} 